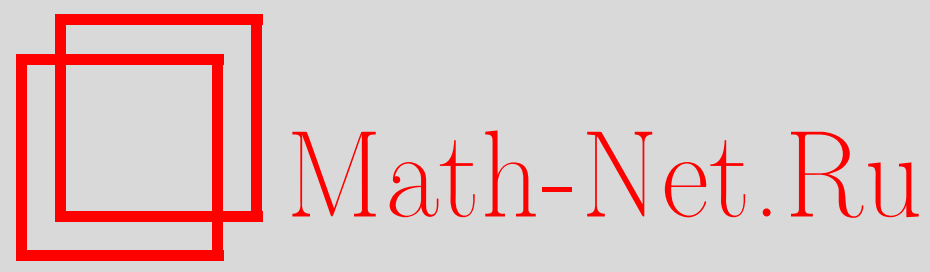

А.-P. К. Рамазанов, О строении пространств полианалитических функций, Матем. заметки, 2002, том 72, выпуск $5,750-764$

DOI: https://doi.org/10.4213/mzm465

Использование Общероссийского математического портала Math-Net.Ru подразумевает, что вы прочитали и согласны с пользовательским соглашением http://www.mathnet.ru/rus/agreement

Параметры загрузки:

IP: 18.207 .199 .55

26 апреля 2023 г., $11: 33: 40$

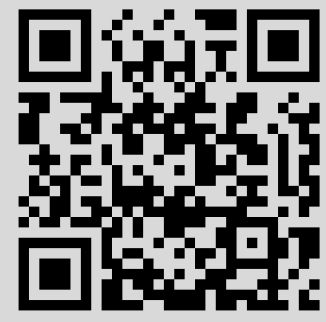


том 72 выпУСК 5 нояБрь 2002

УДК 517.5

\section{О СТРОЕНИИ ПРОСТРАНСТВ \\ ПОЛИАНАЛИТИЧЕСКИХ ФУНКЦИЙ}

\section{А. К. Рамазанов}

Пусть $A_{m} L_{p}(D, \alpha)$-пространство всех $m$-аналитических в круге $D=\{z:|z|<1\}$ функций, суммируемых в $p$-й степени по площади с весом $\left(1-|z|^{2}\right)^{\alpha}, \alpha>-1$. В работе вводятся подпространства $A_{k} L_{p}^{0}(D, \alpha), k=1,2, \ldots, m$, пространства $A_{m} L_{p}(D, \alpha)$ и доказьвается, что $A_{m} L_{p}(D, \alpha)$ является прямой суммой этих подпространств. Эти результаты используются для получения оценок роста производных полианалитических функций вблизи границы произвольных областей.

Библиография: 16 названий.

Функция $f(z)$, которая имеет в области $G$ непрерывные частные производные по $x$ и $y$ до порядка $m \geqslant 1$, назьвается полианалитической функиией (ПАФ) порядка $m$ в области $G$, если в этой области она удовлетворяет обобщенному уравнению Коши-Римана

$$
\frac{\partial^{m} f}{\partial \bar{z}^{m}}=0
$$

Класс таких функций мы будем в дальнейшем обозначать через $A_{m}(G)$.

Известно, что любую ПА $\Phi$ порядка $m$ в области $G$ можно единственным образом представить в виде (см. [1])

$$
f(z)=\varphi_{0}(z)+\bar{z} \varphi_{1}(z)+\cdots+\bar{z}^{m-1} \varphi_{m-1}(z)
$$

где $\varphi_{k}$ голоморфны в $G$. В случае, когда $G=D:=\{z:|z|<1\}$, равенство (1) можно преобразовать к виду (см. [2], [3])

$$
f(z)=P(z, \bar{z})+g_{0}(z)+\left(1-|z|^{2}\right) g_{1}(z)+\cdots+\left(1-|z|^{2}\right)^{m-1} g_{m-1}(z),
$$

где $g_{k}$ голоморфны в $D, P(z, \bar{z})=P_{0}+\bar{z} P_{1}(z)+\cdots+\bar{z}^{m-1} P_{m-1}(z), P_{0}=$ const, $P_{k}(z)$ при $k \geqslant 1-$ полином от $z$ степени не выше $k-1$.

В дальнейшем функции $\varphi_{k}$ и $g_{k}$ будем назьвать голоморфными компонентами $\Pi$ П $\Phi f(z)$.

В работах [2]-[7] исследованы задачи граничного поведения ПА $\Phi$, их формальных производных и голоморфных компонент в интегральных метриках с использованием различных представлений ПАФ. В этой работе мы продолжаем эту тему.

Работа выполнена при поддержке Российского фонда фундаментальных исследований, грант № 99-01-00119. 
Введем необходимые обозначения: $L_{p}(D, \alpha)$ обозначаетпространство функций $f$, для которых конечна норма

$$
\|f\|_{(p, D, \alpha)}=\left\{\iint_{D}\left(1-|z|^{2}\right)^{\alpha}|f(z)|^{p} d x d y\right\}^{1 / p}, \quad 1 \leqslant p \leqslant \infty, \quad \alpha>-1 ;
$$

$A_{k}(D)$ - множество всех функций $k$-аналитических в $D, k=1,2, \ldots, A(D)=A_{1}(D)$; $A_{k} L_{p}(D, \alpha)=A_{k}(D) \cap L_{p}(D, \alpha), A L_{p}(D, \alpha)=A_{1} L_{p}(D, \alpha)$

$$
\begin{aligned}
A_{k}^{0} L_{p}(D, \alpha)= & \left\{f(z)=\left(1-|z|^{2}\right)^{-\alpha} \frac{\partial^{k-1}}{\partial z^{k-1}}\left((1-z \bar{z})^{k-1+\alpha} F(z)\right)\right. \\
& \left.F \in A(D), f \in A_{k} L_{p}(D, \alpha)\right\}
\end{aligned}
$$

Очевидно, что $A_{1} L_{p}(D, \alpha)=A L_{p}(D, \alpha)=A_{1}^{0} L_{p}(D, \alpha)$. При $p=\infty$ считаем везде, что $\alpha \geqslant 0$. Для плоской меры Лебега $d x d y$ также будем использовать обозначение $d \sigma(z)$.

Всюду ниже $\Gamma(\cdot)$ - гамма функция Эйлера; $C_{j}(m, p, \ldots), j=1,2, \ldots,-$ величины, вообще говоря, различные в различных выражениях, зависяшие лишь от указанных в скобках параметров;

$$
\left(\begin{array}{l}
n \\
k
\end{array}\right)=\frac{n !}{k !(n-k) !}, \quad\left(\begin{array}{l}
n \\
k
\end{array}\right)=0 \quad \text { при } k>n \text { и при } k<0 .
$$

Универсальное разложение пространства полианалитических в $D$ функций из $L_{p}(D, \alpha)$ в прямую сумму подпространств.

Tеорема 1. Пусть $1 \leqslant p \leqslant \infty, \alpha>-1$ при $p<\infty, \alpha \geqslant 0$ при $p=\infty$. Функиия

$$
f(z)=\left(1-|z|^{2}\right)^{-\alpha} \frac{\partial^{k-1}}{\partial z^{k-1}}\left((1-z \bar{z})^{k-1+\alpha} F(z)\right)
$$

принадлехст пространству $A_{k} L_{p}(D, \alpha)$ тогда и только тогда, когда $F$ принадлежит $A L_{p}(D, \alpha)$. При этом

$$
\|f\|_{(p, D, \alpha)} \leqslant 2^{2 k-3+2 \alpha / p} \frac{\Gamma(2 k-1+\alpha)}{\Gamma(k+\alpha)}\|F\|_{(p, D, \alpha)} .
$$

ЗАмЕчАниЕ 1. Выражение (4) определяет линейный оператор, отображающий пространство аналитических функций $A L_{p}(D, \alpha)$ в пространство $A_{k}^{0} L_{p}(D, \alpha)$. Неравенство (5) дает оценку нормы этого оператора, а из следствия 2.2 мы получаем оценку нормы его обратного оператора.

Для формулировки следующей теоремы введем еще обозначения:

$$
\begin{gathered}
\mathscr{R}_{k}^{0, \alpha}(z, \zeta, r)=\frac{1}{\pi(k-1) !} \frac{\Gamma(\alpha+1)}{\Gamma(k+\alpha)} \frac{\left(r^{2}-|z|^{2}\right)^{-\alpha}\left(r^{2}-|\zeta|^{2}\right)^{-\alpha}}{r^{2(k-1)}} \\
\times \frac{\partial^{2(k-1)}}{\partial z^{k-1} \partial \bar{\zeta}^{k-1}}\left\{\left(r^{2}-z \bar{z}\right)^{k-1+\alpha}\left(r^{2}-\zeta \bar{\zeta}\right)^{k-1+\alpha} \frac{(k-1)\left(r^{2}-z \bar{\zeta}\right)+r^{2}(\alpha+1)}{\left(r^{2}-z \bar{\zeta}\right)^{\alpha+2}}\right\} \\
\mathscr{R}_{m}^{\alpha}(z, \zeta, r)=\sum_{k=1}^{m} \mathscr{R}_{k}^{0, \alpha}(z, \zeta, r), \quad \mathscr{R}_{k}^{0, \alpha}(z, \zeta, 1)=\mathscr{R}_{k}^{0, \alpha}(z, \zeta), \quad \mathscr{R}_{m}^{\alpha}(z, \zeta, 1)=\mathscr{R}_{m}^{\alpha}(z, \zeta), \\
z, \zeta \in D(0, r), \quad D(t, r)=\{z:|z-t|<r\}, \quad D(0,1)=D .
\end{gathered}
$$


Очевидно, что $\mathscr{R}_{k}^{0, \alpha}(z, \zeta)=\overline{\mathscr{R}_{k}^{0, \alpha}(\zeta, z)}$ и при фиксированном $\zeta \in D$ функция $\mathscr{R}_{k}^{0, \alpha}(z, \zeta)$ принадлежит пространству $A_{k}^{0} L_{p}(D, \alpha)$.

Введем интегральньй оператор

$$
\begin{gathered}
P_{k}^{0, \alpha}(f)(\zeta)=\iint_{D}\left(1-|z|^{2}\right)^{\alpha} f(z) \overline{\mathscr{R}_{k}^{0, \alpha}(z, \zeta)} d x d y, \\
f \in L_{p}(D, \alpha), \quad \zeta \in D, \quad z=x+i y \in D .
\end{gathered}
$$

Оператор, получаемый из формулы (6) при замене ядра $\mathscr{R}_{k}^{0, \alpha}(z, \zeta)$ на ядро $\mathscr{R}_{m}^{\alpha}(z, \zeta)$, будем обозначать через $P_{m}^{\alpha}$.

Очевидно, что $P_{m}^{\alpha}=\sum_{k=1}^{m} P_{k}^{0, \alpha}$.

Теорема 2. 1. Оператор $P_{k}^{0, \alpha}\left(P_{m}^{\alpha}\right)$ является оператором проектирования пространства $L_{p}(D, \alpha)$ на его подпространство $A_{k}^{0} L_{p}(D, \alpha)$ (соответственно на $\left.A_{m} L_{p}(D, \alpha)\right)$ nрu $1<p<\infty$ в всех $\alpha>-1$.

2. При $p=2$ эти операторы являются ортогональными проекторами на соответствующие подпространства.

3. Если $f \in A_{k}^{0} L_{p}(D, \alpha)\left(f \in A_{m} L_{p}(D, \alpha)\right)$, mo $P_{k}^{0, \alpha}(f)(z)=f(z), z \in D$ (coomветственно $\left.P_{m}^{\alpha}(f)(z)=f(z), z \in D\right)$, при всех $1 \leqslant p \leqslant \infty$.

СлЕДСТВИЕ 2.1. Пусть $f \in A_{m} L_{p}(D, \alpha) u \varphi_{j}(z)$ - голоморфные компоненты функиии $f$ в представлении (1). Тогда

$$
\begin{array}{ll}
\varphi_{j} \in A L_{p}(D, \alpha+p(m-1)) & n p u \quad 1 \leqslant p<\infty \quad u \quad \alpha>-1, \\
\varphi_{j} \in A L_{p}(D, \alpha+(m-1)) & n p u \quad p=\infty \quad u \quad \alpha \geqslant 0, j=0,1, \ldots, m-1 .
\end{array}
$$

СЛЕДСТВИЕ 2.2. Если ПАФ

$$
f(z)=\left(1-|z|^{2}\right)^{-\alpha} \frac{\partial^{k-1}}{\partial z^{k-1}}\left((1-z \bar{z})^{k-1+\alpha} F(z)\right)
$$

принадлежст пространству $A_{k}^{0} L_{p}(D, \alpha), 1<p<\infty$, то

$$
C_{1}(k, p, \alpha)\|F\|_{(p, D, \alpha)} \leqslant\|f\|_{(p, D, \alpha)} \leqslant C_{2}(k, p, \alpha)\|F\|_{(p, D, \alpha)} .
$$

ТЕорема 3. При $1 \leqslant p \leqslant \infty$ пространство $A_{m} L_{p}(D, \alpha)$ является прямой суммой подпространств $A_{k}^{0} L_{p}(D, \alpha)$ с $k=1,2, \ldots, m$ :

$$
A_{m} L_{p}(D, \alpha)=A_{1} L_{p}(D, \alpha) \oplus A_{2}^{0} L_{p}(D, \alpha) \oplus \cdots \oplus A_{m}^{0} L_{p}(D, \alpha)
$$

ЗАмечаниЕ 2. Теоремы 1,2 и 3 при $p=2$ получены в [5], [6]. 
ЗАмЕчАниЕ 3 . Легко видеть, что при $m=1$ ядро $\mathscr{R}_{m}^{\alpha}(z, \zeta)$ совпадает с известным ядром Бергмана для круга $D$. Интегральные операторы вида (6) с ядром Бергмана исследованы в работах [8]-[11]. Операторы вида (6) для ПАФ в метрике пространства $L_{p}(D)$ при $1 \leqslant p<\infty$ исследованы также в работе [11].

В [11] приводится (без доказательства) другое представление для ядра $\mathscr{R}_{m}^{\alpha}(z, \zeta)$ в случае $\alpha=0$ :

$$
\mathscr{R}_{m}(z, \zeta)=\frac{m}{\pi(1-z \bar{\zeta})^{2 m}} \sum_{j=0}^{m-1}(-1)^{j}\left(\begin{array}{c}
m \\
j+1
\end{array}\right)\left(\begin{array}{c}
m+j \\
m
\end{array}\right)|1-z \bar{\zeta}|^{2(m-1-j)}|z-\zeta|^{2 j}
$$

Теоремы 1-3 можно использовать для получения оценок роста ПАФ при стремлении точки $z$ к границе области, а также для исследования полирациональных приближений в метрике (3) (см. [6], [13]). Этим вопросам посвящена вторая часть настоящей работы.

Для доказательства сформулированных теорем нужны вспомогательные утверждения.

ОПРЕДЕЛЕНИЕ. Односвязную ограниченную область $G$ с гладкой жордановой гранищей $\partial G$ будем назьвать обобщенно ляпуновской, если модуль непрерывности $\omega(\delta)$ угла наклона касательной к $\partial G$ как функции длины $s$ дуги удовлетворяет условию Дини

$$
\int_{0} \frac{\omega(t)}{t} d t<\infty
$$

Для произвольной области $G$ пространства $L_{p}(G, \alpha)$ определяются путем замены веса $\left(1-|z|^{2}\right)^{\alpha}$ в равенстве (3) на весовую функцию $(\rho(z))^{\alpha}$, где $\rho(z)=\rho(z, \partial G)$. Как и выше, $A_{m} L_{p}(G, \alpha)=A_{m}(G) \cap L_{p}(G, \alpha)$.

Если $f \in A_{m}(G)$, то

$$
\partial^{s} f(z):=\frac{\partial^{s} f(z)}{\partial \bar{z}^{n} \partial z^{s-n}}, \quad n=0,1, \ldots, s, \quad n<m, \quad s=0,1, \ldots
$$

ЛЕмма 1 [4]. Пусть $G$ - обобщенно ляпуновская область. Тогда для любой функиии $\Phi$, голоморфной в области $G$, имеем

$$
\begin{aligned}
& \Phi \in A L_{p}(G, \alpha) \Longleftrightarrow \Phi^{(s)} \in A L_{p}(G, \alpha+p s) \quad \text { npu } 1 \leqslant p<\infty u \alpha>-1, \\
& \Phi \in A L_{p}(G, \alpha) \Longleftrightarrow \Phi^{(s)} \in A L_{p}(G, \alpha+s) \quad \text { npu } p=\infty u \alpha \geqslant 0 .
\end{aligned}
$$

Лемма 2 [4]. Пусть $G$ - произвольная область. Тогда для любой функиии $f \in$ $A_{m}(G)$ имеем

$$
\begin{array}{ll}
f \in A_{m} L_{p}(G, \alpha) \Rightarrow \partial^{s} f \in A_{m} L_{p}(G, \alpha+s p) & \text { npu } 1 \leqslant p<\infty u \alpha>-1, \\
f \in A_{m} L_{p}(G, \alpha) \Rightarrow \partial^{s} f \in A_{m} L_{p}(G, \alpha+s) & \text { npu } p=\infty u \alpha \geqslant 0 .
\end{array}
$$

Леммы 1 и 2 являются следствиями более общих результатов работы [4] (см. теорему 5 и следствие Т5 из нее, а также промежуточное неравенство, полученное на с. 23). Лемма 1 в указанном виде обобщает результаты Харди-Литтлвуда и А. Забулениса, полученные ими для круга (см. [14, с. 81] и [15]), на области с обобщенно ляпуновской гранищей, а лемма 2 переносит эти результаты на полианалитические функции. Отметим также, что для обобщенно ляпуновских областей в работе [4] доказана и обратная импликация леммы 2. 
ЛЕмма 3. Для любых натуральных чисел $k, n$ и любого действительного числа $\alpha>-1$ справедливо равенство

$$
\sum_{j=0}^{k-1}\left(\begin{array}{c}
k-1 \\
j
\end{array}\right)\left(\begin{array}{l}
n \\
j
\end{array}\right) j ! \frac{\Gamma(k+\alpha)}{\Gamma(j+1+\alpha)}=\frac{\Gamma(n+k+\alpha)}{\Gamma(n+1+\alpha)}
$$

ДокАЗАТЕЛЬСТво. Для $t \geqslant 0$ рассмотрим функцию $t^{n} t^{k-1+\alpha}$. Используя правило Лейбница, найдем ее производную:

$$
\left(t^{n} t^{k-1+\alpha}\right)^{(k-1)}=\sum_{j=0}^{k-1}\left(\begin{array}{c}
k-1 \\
j
\end{array}\right)\left(\begin{array}{c}
n \\
j
\end{array}\right) j ! \frac{\Gamma(k+\alpha)}{\Gamma(j+1+\alpha)} t^{n+\alpha} .
$$

Вычислив производную в правой части равенства (8) как производную степенной функции $t^{n+k-1+\alpha}$, мы получим требуемый результат.

ЛЕмма 4. Пусть $\alpha>-1 u z, \zeta \in D$. Тогда

$$
\iint_{D} \frac{\left(1-|z|^{2}\right)^{\alpha}}{|1-\zeta \bar{z}|^{2+\alpha+\beta}} d x d y \leqslant \begin{cases}C_{1}(\alpha, \beta)\left(1-|\zeta|^{2}\right)^{-\beta}, & \text { если } \beta>0 \\ C_{2}(\alpha, \beta), & \text { если } \beta<0\end{cases}
$$

Доказательство леммы 4 имеется в [16, гл. 1, с. 26].

Лемма 5. Для любых $z, \zeta \in D$ справедливы следующие неравенства:

$$
\begin{aligned}
& \text { 1. } \quad\left|\mathscr{R}_{k}^{0, \alpha}(z, \zeta)\right| \leqslant \frac{C_{1}(\alpha, k)}{|1-z \bar{\zeta}|^{2+\alpha}}, \quad k=1,2, \ldots, m, \quad z, \zeta \in D ; \\
& \text { 2. } \quad\left|\mathscr{R}_{m}^{\alpha}(z, \zeta)\right| \leqslant \frac{C_{2}(\alpha, m)}{|1-z \bar{\zeta}|^{2+\alpha}}, \quad z, \zeta \in D .
\end{aligned}
$$

ЗАмЕчаниЕ 4. Приведенная оценка для ядра $\mathscr{R}_{m}^{\alpha}(z, \zeta)$ получена в [11]. Приводимое ниже доказательство является существенно более простым, поскольку использует явный вид ядра $\mathscr{R}_{m}^{\alpha}(z, \zeta)$.

ДоКАЗАТЕЛЬСТВО ЛЕмМЫ 5. Путем несложных преобразований нетрудно получить следующую оценку для ядра $\mathscr{R}_{k}^{0, \alpha}(z, \zeta)$ :

$$
\left|\mathscr{R}_{k}^{0, \alpha}(z, \zeta)\right| \leqslant \frac{C(\alpha, k)}{|1-z \bar{\zeta}|^{2+\alpha}} \sum_{m, n=0}^{k} \frac{\left(1-|z|^{2}\right)^{m}\left(1-|\zeta|^{2}\right)^{n}}{|1-z \bar{\zeta}|^{m+n}}
$$

Легко заметить, что

$$
|1-z \bar{\zeta}|^{2}=\left(1-|z|^{2}\right)\left(1-|\zeta|^{2}\right)+|z-\zeta|^{2}
$$

Далее, любые две точки $z, \zeta \in D$ удовлетворяют одному из следующих условий: 
1)

$$
\left\{\begin{array}{l}
|z-\zeta| \geqslant \frac{1}{4}\left(1-|\zeta|^{2}\right) \\
|z-\zeta| \geqslant \frac{1}{4}\left(1-|z|^{2}\right)
\end{array}\right.
$$

2) хотя бы в одном из неравенств условия 1) вьполняется противоположное неравенство.

Если для точек $z, \zeta \in D$ вьполняется условие 1$)$, то, учитывая равенство (10), получаем

$$
\frac{\left(1-|z|^{2}\right)^{m}\left(1-|\zeta|^{2}\right)^{n}}{|1-z \bar{\zeta}|^{m+n}} \leqslant \frac{4^{m+n}|z-\zeta|^{m+n}}{|z-\zeta|^{m+n}} \leqslant 4^{m+n} .
$$

Пусть теперь выполняется условие 2$)$ и допустим, что $|z-\zeta|<1 / 4\left(1-|z|^{2}\right)$. Тогда $\left(1-|z|^{2}\right)$ и $\left(1-|\zeta|^{2}\right)$ слабо эквивалентны. Действительно,

$$
\frac{1}{4}\left(1-|z|^{2}\right)<\left(1-|\zeta|^{2}\right)<2(|z-\zeta|+(1-|z|))<2\left(\frac{1}{4}\left(1-|z|^{2}\right)+\left(1-|z|^{2}\right)\right)=\frac{5}{2}\left(1-|z|^{2}\right) .
$$

Из равенства (10) тогда следует

$$
|1-z \bar{\zeta}|^{2} \geqslant\left(1-|z|^{2}\right)\left(1-|\zeta|^{2}\right) \geqslant \frac{1}{4}\left(1-|z|^{2}\right)^{2} .
$$

Следовательно, и в этом случае

$$
\frac{\left(1-|z|^{2}\right)^{m}\left(1-|\zeta|^{2}\right)^{n}}{|1-z \bar{\zeta}|^{m+n}} \leqslant \frac{\left.\left.|1-| z\right|^{2}\right)^{m}(5 / 2)^{n}\left(1-|z|^{2}\right)^{n}}{(1 / 2)^{m+n}\left(1-|z|^{2}\right)^{m+n}} \leqslant 2^{m} 5^{n}
$$

Из неравенств (9), (11), (12) получаем требуемьй результат.

ДоКАЗАТЕЛЬСТво ТЕОРЕМЫ 1. Представим функцию $f$ в виде

$$
f(z)=\sum_{j=0}^{k-1}(-1)^{k-1-j}\left(\begin{array}{c}
k-1 \\
j
\end{array}\right) \frac{\Gamma(k+\alpha)}{\Gamma(\alpha+j+1)}\left(1-|z|^{2}\right)^{j} F^{(j)}(z) \bar{z}^{k-1-j} .
$$

Тогда

$$
\begin{aligned}
\frac{\partial^{k-1} f(z)}{\partial \bar{z}^{k-1}} & =(-1)^{k-1} \Gamma(k) \sum_{j=0}^{k-1}\left(\begin{array}{c}
k-1 \\
j
\end{array}\right) \frac{\Gamma(k+\alpha)}{\Gamma(\alpha+j+1)} F^{(j)}(z) z^{j} \\
& =\Gamma(k)(-1)^{k-1} z^{-\alpha}\left(z^{k-1+\alpha} F(z)\right)^{(k-1)}
\end{aligned}
$$

Из леммы 2 следует, что функция $\partial^{k-1} f / \partial \bar{z}^{k-1}$ принадлежит пространству $A_{k} L_{p}(D$, $\alpha+(k-1) p)$.

Так как $\partial^{k-1} f / \partial \bar{z}^{k-1}$ аналитична в $D$, применив лемму 1 , легко получаем, что $F \in$ $A L_{p}(D, \alpha)$.

Докажем теперь обратное утверждение. Пусть $F \in A L_{p}(D, \alpha)$. Тогда из леммы 1 следует, что $F^{(j)} \in A L_{p}(D, \alpha+j p)$. Далее, воспользовавшись равенством (13), получаем, что $f \in A_{m} L_{p}(D, \alpha)$. 
Остается доказать неравенство (5). Пусть $z$ - произвольная точка круга $D$. Рассмотрим окружность $C_{\rho}$ радиуса $\rho$ с центром в точке $z$ так, чтобы $C_{\rho} \subset D$. Тогда по интегральной формуле Коши имеем

$$
F^{(j)}(z)=\frac{j !}{2 \pi i} \int_{C_{\rho}} \frac{F(\zeta)}{(\zeta-z)^{j+1}} d \zeta
$$

Положив $\zeta-z=\left(1-|z|^{2}\right) e^{i \varphi} / 4$, получаем

$$
\left(1-|z|^{2}\right)^{j} F^{(j)}(z)=\frac{j ! 4^{j}}{2 \pi} \int_{0}^{2 \pi} F(\zeta) e^{-i j \varphi} d \varphi .
$$

Учитывая представление (13) для функции $f$, из равенства (14) легко следует, что

$$
f(z)=\frac{1}{\pi} \sum_{j=0}^{k-1}(-1)^{k-1-j}\left(\begin{array}{c}
k-1 \\
j
\end{array}\right) \frac{\Gamma(k+\alpha)}{\Gamma(\alpha+j+1)} \bar{z}^{k-1-j} j ! 2^{2 j-1} \int_{0}^{2 \pi} F(\zeta) e^{-i j \varphi} d \varphi
$$

Так как $j ! \leqslant(k-1) ! /((k-1-j) !)$, используя лемму 3 при $n=k-1$, из последнего равенства получаем

$$
|f(z)| \leqslant \frac{2^{2 k-3}}{\pi} \frac{\Gamma(2 k-1+\alpha)}{\Gamma(k+\alpha)} \int_{0}^{2 \pi}|F(\zeta)| d \varphi .
$$

Очевидно, что при $|z-\zeta| \leqslant\left(1-|z|^{2}\right) / 4, z \in D$, выполняется неравенство $1-|z|^{2} \leqslant$ $4\left(1-|\zeta|^{2}\right)$. Поэтому, проинтегрировав неравенство (15) по кругу $D$ и применив обобщенное неравенство Минковского, получаем

$$
\|f\|_{(p, D, \alpha)} \leqslant 2^{2 k-3+2 \alpha / p} \frac{\Gamma(2 k-1+\alpha)}{\Gamma(k+\alpha)}\left\{\iint_{D}\left(1-|z|^{2}\right)^{\alpha}|F(z)|^{p} d x d y\right\}^{1 / p} .
$$

ДоКАЗАТЕЛЬСТВО ТЕОРЕМЫ 2. При $p=2$ теорема доказана в [5], [6]. Легковидеть, что оператор $P_{k}^{0, \alpha}$ определен для любой функции $f \in L_{p}(D, \alpha), 1 \leqslant p \leqslant \infty$, и

$$
P_{k}^{0, \alpha}(f)(z)=\left(1-|z|^{2}\right)^{-\alpha} \frac{\partial^{k-1}}{\partial z^{k-1}}\left((1-z \bar{z})^{k-1+\alpha} F(z)\right), \quad \text { где } F \in A(D) .
$$

Докажем ограниченность этого оператора при $1<p<\infty$. Пусть

$$
\beta= \begin{cases}\frac{1}{p q}, & \text { если } \alpha \geqslant 0, \\ \frac{\alpha+1}{p q}, & \text { если }-1<\alpha<0,\end{cases}
$$

где $1 / p+1 / q=1$. Тогда, как и в работе [16, с. 131], рассмотрев функцию $h(z)=\left(1-|z|^{2}\right)^{-\beta}$ и используя леммы 4 и 5 , доказываем ограниченность оператора $P_{k}^{0, \alpha}$ в пространстве $L_{p}(D, \alpha), 1<p<\infty$.

Далее, пусть $f \in A_{k}^{0} L_{p}(D, \alpha) \cap A_{k}^{0} L_{2}(D, \alpha), 1 \leqslant p \leqslant \infty$. Тогда по п. 2 доказываемой теоремы имеем

$$
P_{k}^{0, \alpha}(f)(z)=f(z), \quad z \in D .
$$

Так как множество $A_{k}^{0} L_{p}(D, \alpha) \cap A_{k}^{0} L_{2}(D, \alpha)$ всюду плотно в пространстве $A_{k}^{0} L_{p}(D$, $\alpha), 1 \leqslant p \leqslant \infty$, то равенство (16) справедливо для любой функции $f \in A_{k}^{0} L_{p}(D, \alpha)$, $1 \leqslant p \leqslant \infty$.

Оператор $P_{m}^{\alpha}$ рассматривается аналогично.

Следствие 2.1 доказывается по аналогии с доказательством следствия 2 из [6]. 
ДоКАЗАТЕЛЬСТво СЛЕДСТвИЯ 2.2. Неравенство

$$
\|f\|_{(p, D, \alpha)} \leqslant C_{2}(k, p, \alpha)\|F\|_{(p, D, \alpha)}
$$

доказано в теореме 1 с константой $C_{2}(k, p, \alpha)=2^{2 k-3+2 \alpha / p} \Gamma(2 k-1+\alpha) / \Gamma(k+\alpha)$ при всех $p$, удовлетворяюших неравенству $1 \leqslant p \leqslant \infty$. Докажем левое неравенство.

Из теоремы 2 имеем

$$
P_{k}^{0, \alpha}(f)(z)=f(z) \quad \text { для любой функции } f \in A_{k}^{0} L_{p}(D, \alpha) .
$$

Тогда, используя интегральное представление (6), получаем (см. еще $[6]$ при $\alpha=0$ )

$$
F(\zeta)=\iint_{D}\left(1-|z|^{2}\right)^{\alpha} f(z) \overline{T_{k}^{0, \alpha}(z, \zeta)} d x d y
$$

где

$$
T_{k}^{0, \alpha}(z, \zeta)=\frac{1}{\pi} \frac{\Gamma(\alpha+1)\left(1-|z|^{2}\right)^{-\alpha}}{\Gamma(k) \Gamma(k+\alpha)} \frac{\partial^{k-1}}{\partial z^{k-1}}\left((1-z \bar{z})^{k-1+\alpha} \frac{(k-1)(1-z \bar{\zeta})+\alpha+1}{(1-z \bar{\zeta})^{\alpha+2}}\right) .
$$

Как и при доказательстве леммы 5, устанавливается, что

$$
\left|T_{k}^{0, \alpha}(z, \zeta)\right| \leqslant \frac{C(k, \alpha)}{|1-z \bar{\zeta}|^{\alpha+2}}, \quad z, \zeta \in D
$$

Используя метод доказательства теоремы 2 с той же функцией $h(z)=\left(1-|z|^{2}\right)^{-\beta}$, завершаем доказательство следствия 2.2 .

ДоКАЗАТЕЛЬСТво тЕОРЕмЫ 3 . При $1<p<\infty$ утверждение теоремы 3 получаем из теоремы 2. Действительно, если $f \in A_{m} L_{p}(D, \alpha), 1<p<\infty$, то, применив оператор $P_{m}^{\alpha}$, получаем

$$
f(z)=P_{m}^{\alpha}(f)(z)=\sum_{k=1}^{m} P_{k}^{0, \alpha}(f)(z),
$$

где $P_{k}^{0, \alpha}(f) \in A_{k}^{0} L_{p}(D, \alpha), k=1,2, \ldots, m$.

Если же $p=\infty$, то для любой функции $f \in A_{m} L_{p}(D, \alpha)$ имеем

$$
f(z)=P_{m}^{\alpha}(f)(z)=\sum_{k=1}^{m} P_{k}^{0, \alpha}(f)(z)=\left(1-|z|^{2}\right)^{-\alpha} \sum_{k=1}^{m} \frac{\partial^{k-1}}{\partial z^{k-1}}\left((1-z \bar{z})^{k-1+\alpha} F_{k}(z)\right),
$$

где $F_{k} \in A(D)$. Докажем, что $F_{k} \in A L_{\infty}(D, \alpha), k=1,2, \ldots, m$. Тогда из теоремы 1 будет следовать, что $P_{k}^{0, \alpha}(f) \in A_{k}^{0} L_{\infty}(D, \alpha), \alpha \geqslant 0$, при $k=1,2, \ldots, m$.

Как и при доказательстве теоремы 1 , находим

$$
\frac{\partial^{m-1} f(z)}{\partial \bar{z}^{m-1}}=\Gamma(m)(-1)^{m-1} z^{-\alpha}\left(z^{m-1+\alpha} F(z)\right)^{(m-1)} .
$$

По лемме 2 функция $\partial^{m-1} f / \partial \bar{z}^{m-1}$ принадлежит пространству $A_{m} L_{\infty}(D, \alpha+m-1)$. Так как $\partial^{m-1} f / \partial \bar{z}^{m-1}$ является аналитической функцией в $D$, применив лемму 1 , получаем, что $F_{m} \in A L_{\infty}(D, \alpha)$. 
Таким образом, $P_{m}^{0, \alpha}(f) \in A_{m}^{0} L_{\infty}(D, \alpha)$.

Воспользуемся методом математической индукции. Пусть мы доказали, что $P_{j}^{0, \alpha}(f)$ $\in A_{j}^{0} L_{\infty}(D, \alpha)$ при $j=m, m-1, \ldots, k+1$.

Рассмотрим функцию

$$
g(z)=f(z)-\sum_{j=k+1}^{m} P_{j}^{0, \alpha}(f)(z)=\sum_{j=1}^{k} P_{j}^{0, \alpha}(f)(z),
$$

которая принадлежит пространству $A_{k} L_{\infty}(D, \alpha)$. Как и выше, вычислив производную $\partial^{k-1} g / \partial \bar{z}^{k-1}$, доказьваем, что $F_{k} \in A L_{\infty}(D, \alpha)$. Тогда по теореме $1 P_{k}^{0, \alpha}(f) \in$ $A_{k}^{0} L_{\infty}(D, \alpha)$, что и требовалось доказать.

Пусть теперь $p=1$. Тогда из теоремы 2 следует, что для любой функции $f \in$ $A_{m} L_{1}(D, \alpha)$

$$
f(z)=P_{m}^{\alpha}(f)(z)=\sum_{k=1}^{m} P_{k}^{0, \alpha}(f)(z)=\left(1-|z|^{2}\right)^{-\alpha} \sum_{k=1}^{m} \frac{\partial^{k-1}}{\partial z^{k-1}}\left((1-z \bar{z})^{k-1+\alpha} F_{k}(z)\right),
$$

где $F_{k} \in A(D)$. Как и в случае $p=\infty$, доказываем сначала, что $F_{k} \in A L_{1}(D, \alpha)$, $k=1,2, \ldots, m$, а потом, применив теорему 1 , завершаем доказательство теоремы 3 и при $p=1$.

Оценки производных полианалитических функций. Для произвольной области $G$ из $\bar{C}$ в качестве весовой функции будем рассматривать следующую функцию: $(\rho(z))^{\alpha}=(\rho(z, \partial G))^{\alpha}, \alpha>-1$, где $\rho(z)=\rho(z, \partial G)$ - расстояние от $z \in G$ до границы $\partial G$.

Пусть $L_{p}(G, \alpha), \alpha>-1,1 \leqslant p \leqslant \infty$, обозначает пространство комплекснозначных функций $f$ с нормой

$$
\|f\|_{(p, G, \alpha)}=\left\{\iint_{G}(\rho(z))^{\alpha}|f(z)|^{p} d x d y\right\}^{1 / p}<\infty .
$$

Очевидно, что при $D=G$ нормы, определяемые равенствами (3) и (17), эквивалентны. Поэтому мы сохраняем наши старые обозначения и для произвольной области $G$. В частности, $L_{p}(G, 0)=L_{p}(G), A_{m} L_{p}(G, \alpha)=A_{m}(G) \cap L_{p}(G, \alpha)$.

Всюду ниже $p$ и $q$ - сопряженные числа, т.е. $1 \leqslant p \leqslant \infty, 1 \leqslant q \leqslant \infty$ и $1 / p+1 / q=1$.

Введем еще обозначения:

$$
\begin{gathered}
A_{1}(k, s, p, \alpha)=\left\{\int_{0}^{1}(1-t)^{\alpha}\left|t^{-s / 2}(1-t)^{-\alpha}\left(t^{k-1+s}(1-t)^{k-1+\alpha}\right)^{(k-1)}\right|^{q} d t\right\}^{1 / q} \\
\text { при } 1<p \leqslant \infty \text { и } s=0,1, \ldots, \\
A_{2}(k, s, p, \alpha)=\left\{\int_{0}^{1}(1-t)^{\alpha}\left|t^{s / 2}(1-t)^{-\alpha}\left(t^{k-1-s}(1-t)^{k-1+\alpha}\right)^{(k-1)}\right|^{q} d t\right\}^{1 / q} \\
\text { при } 1<p \leqslant \infty \text { и } s=0,1, \ldots, k-1, \\
A_{3}(k, s, \alpha)=\max _{0 \leqslant t \leqslant 1}\left\{\left|t^{-s / 2}(1-t)^{-\alpha}\left(t^{k-1+s}(1-t)^{k-1+\alpha}\right)^{(k-1)}\right|\right\} \quad \text { при } s=0,1, \ldots, \\
A_{4}(k, s, \alpha)=\max _{0 \leqslant t \leqslant 1}\left\{\left|t^{s / 2}(1-t)^{-\alpha}\left(t^{k-1-s}(1-t)^{k-1+\alpha}\right)^{(k-1)}\right|\right\} \\
\text { при } s=0,1, \ldots, k-1 .
\end{gathered}
$$

Справедлива следуюшая теорема. 
ТЕорема 4. Пусть $f \in A_{m} L_{p}(G, \alpha), 1 \leqslant p \leqslant \infty$, где $G$ - произвольная область на $\bar{C}$. Тогда

$$
\begin{array}{ll}
\text { 1) } \quad\left|\frac{\partial^{s} f(z)}{\partial z^{s}}\right| \leqslant C_{1}(m, s, p, \alpha) \rho^{-(2 \alpha+2+s p) / p}\|f\|_{(p, D(z, \rho), \alpha)}, & s=0,1, \ldots, \\
\text { 2) } \quad\left|\frac{\partial^{s} f(z)}{\partial \bar{z}^{s}}\right| \leqslant C_{2}(m, s, p, \alpha) \rho^{-(2 \alpha+2+s p) / p}\|f\|_{(p, D(z, \rho), \alpha)}, & s=0,1, \ldots, m-1,
\end{array}
$$

əде

$$
\begin{aligned}
& C_{1}(m, s, p, \alpha)=\frac{1}{\pi^{1 / p}} \sum_{k=1}^{m} \frac{\Gamma(k+s+\alpha)(2 k-1+s+\alpha)}{\Gamma(k) \Gamma(k+\alpha)} A_{1}(k, s, p, \alpha), \\
& C_{2}(m, s, p, \alpha)=\frac{1}{\pi^{1 / p}} \sum_{k=s+1}^{m} \frac{(2 k-1-s+\alpha)}{\Gamma(k-s)} A_{2}(k, s, p, \alpha) .
\end{aligned}
$$

Теорема 4 при $\alpha=0$ и $p>2$ с другими постоянными получена в [4].

СлЕДСтвие 4.1. Пусть $f \in A L_{p}(G, \alpha), 1 \leqslant p \leqslant \infty$. Тогда

$$
\left|f^{(s)}(z)\right| \leqslant C_{1}(s, p, \alpha) \rho^{-(2 \alpha+2+s p) / p}\|f\|_{(p, D(z, \rho), \alpha)}, \quad s=0,1, \ldots,
$$

əдe

$$
C_{1}(s, p, \alpha)= \begin{cases}\frac{1}{\pi^{1 / p}} \frac{\Gamma(s+\alpha+2)\left(\Gamma\left(\frac{s q}{2}+1\right)\right)^{1 / q}}{(\Gamma(\alpha+1))^{1 / p}\left(\Gamma\left(\frac{s q}{2}+\alpha+2\right)\right)^{1 / q}} & \text { nрu } 1<p \leqslant \infty, \\ \frac{1}{\pi} \frac{\Gamma(s+\alpha+2)}{\Gamma(\alpha+1)} & \text { nри } p=1 .\end{cases}
$$

СлЕДСтвиЕ 4.2. Пусть $f \in A_{m} L_{p}(G, \alpha), 1 \leqslant p \leqslant \infty$. Тогда для компоненты $\varphi_{m-1}$ функиии $f$ в представлении (1) имеем

$$
\left|\varphi_{m-1}(z)\right| \leqslant C_{2}(m, p, \alpha) \rho^{-(2 \alpha+2+(m-1) p) / p}\|f\|_{(p, D(z, \rho), \alpha)},
$$

əде

$$
C_{2}(m, p, \alpha)= \begin{cases}\frac{1}{\pi^{1 / p}} \frac{\Gamma(m+\alpha+1)\left(\Gamma\left(\frac{(m-1) q}{2}+1\right)\right)^{1 / q}}{\Gamma(m)(\Gamma(\alpha+1))^{1 / p}\left(\Gamma\left(\frac{(m-1) q}{2}+\alpha+2\right)\right)^{1 / q}} & \text { npu } 1<p \leqslant \infty, \\ \frac{1}{\pi} \frac{\Gamma(m+\alpha+1)}{\Gamma(\alpha+1)} & \text { nрu } p=1 .\end{cases}
$$

Аналогичные оценки можно получить и для других компонент функции $f$.

ЗАмЕчаниЕ 5. В теореме 4 и следствиях 4.1 и 4.2 при $p=\infty$ считаем, что $\alpha=0$. При $p=1$ в выражениях для $C_{1}(m, s, p, \alpha)$ и $C_{2}(m, s, p, \alpha)$ из теоремы 4 величины $A_{1}(k, s, p, \alpha)$ и $A_{2}(k, s, p, \alpha)$ следует заменить на величины $A_{3}(k, s, \alpha)$ и $A_{4}(k, s, \alpha)$ соответственно.

Для $f \in L_{p}(G)$ положим

$$
\Omega(G, p, f, \delta)=\sup \left\{\|f(\cdot)-f(z)\|_{(p, D(z, r))}: 0<r \leqslant \delta, D(z, r) \subset G\right\}
$$

и $\Omega(G, p, f, 0):=0$. 
ОПРЕДЕЛЕНИЕ. Будем говорить, что $f \in \operatorname{Lip}(\beta, p, G), \beta>0$, если $\Omega(G, p, f, \delta)=$ $O\left(\delta^{\beta}\right)$ при $\delta \rightarrow 0$.

Следующая теорема является обобщением теоремы Харди-Литтлвуда, полученная при $p=\infty$ и $G=D$.

Tеорема 5. Пусть $1 \leqslant p \leqslant \infty, f \in A_{n}(G) u 1 / p<\beta \leqslant 1+2 / p$. Тогдa

$$
f \in \operatorname{Lip}(\beta, p, G) \Longleftrightarrow\left|\frac{\partial f(z)}{\partial z}\right|+\left|\frac{\partial f(z)}{\partial \bar{z}}\right|=O\left(\rho^{\beta-1-2 / p}\right) \quad \text { npu } \rho=\rho(z, \partial G) \rightarrow 0 .
$$

При $2<p \leqslant \infty$ и $1 /(2 p)<\beta \leqslant 1+2 / p, \beta \neq 1 / p, 2 / p$, для ограниченных односвязных областей $G$ с дважды гладкой гранищей теорема 5 получена в [4].

Для доказательства сформулированных теорем нужны вспомогательные утверждения.

Положим

$$
\psi_{1}^{(s)}(z)=\left.\frac{\partial^{s}}{\partial \zeta^{s}}\left(\overline{\mathscr{R}_{k}^{0, \alpha}(z, \zeta, R)}\right)\right|_{\zeta=0}, \quad \psi_{2}^{(s)}(z)=\left.\frac{\partial^{s}}{\partial \bar{\zeta}^{s}}\left(\overline{\mathscr{R}_{k}^{0, \alpha}(z, \zeta, R)}\right)\right|_{\zeta=0} .
$$

ЛЕмма 6. Пусть $1 \leqslant p \leqslant \infty u \alpha>-1$. Тогда имеем:

1) $n p u 1 \leqslant q<\infty$

$$
\begin{aligned}
&\left\|\psi_{1}^{(s)}\right\|_{(q, D(0, R), \alpha)}= \frac{1}{\pi^{1 / p}} \frac{\Gamma(k+s+\alpha)(2 k-1+s+\alpha)}{\Gamma(k) \Gamma(k+\alpha)} R^{-(2(\alpha+1)+p s) / p} A_{1}(k, s, p, \alpha), \\
& s=0,1, \ldots, \\
&\left\|\psi_{2}^{(s)}\right\|_{(q, D(0, R), \alpha)}=\frac{1}{\pi^{1 / p}} \frac{(2 k-1-s+\alpha)}{\Gamma(k-s)} R^{-(2(\alpha+1)+p s) / p} A_{2}(k, s, p, \alpha), \\
& s=0,1, \ldots, k-1 ;
\end{aligned}
$$

2) $n p u q=\infty$

$$
\begin{gathered}
\left\|\psi_{1}^{(s)}\right\|_{(\infty, D(0, R), 0)}=\frac{1}{\pi} \frac{\Gamma(k+s+\alpha)(2 k-1+s+\alpha)}{\Gamma(k) \Gamma(k+\alpha)} R^{-(2(\alpha+1)+s)} A_{3}(k, s, \alpha), \\
s=0,1, \ldots, \\
\left\|\psi_{2}^{(s)}\right\|_{(\infty, D(0, R), 0)}=\frac{1}{\pi} \frac{(2 k-1-s+\alpha)}{\Gamma(k-s)} R^{-(2(\alpha+1)+s)} A_{4}(k, s, \alpha), \\
s=0,1, \ldots, k-1 .
\end{gathered}
$$

ДокАЗАТЕльСТво. Преобразуем ядро $\mathscr{R}_{k}^{0, \alpha}(z, \zeta, R)$, используя его разложениев ряд Фурье по системе полимногочленов

$$
\begin{aligned}
e_{k, n}(z)= & \frac{1}{\sqrt{\pi}} \sqrt{\frac{\Gamma(n+\alpha+1)(k+n+\alpha)}{\Gamma(k) \Gamma(k+\alpha) \Gamma(n+1)}} R^{-(k+n+\alpha)}\left(R^{2}-|z|^{2}\right)^{-\alpha} \\
& \times \frac{\partial^{k-1}}{\partial z^{k-1}}\left\{\left(R^{2}-z \bar{z}\right)^{k-1+\alpha} z^{n}\right\}
\end{aligned}
$$


где $n=0,1, \ldots$ (см. [5], [6]).

Имеем

$$
\begin{aligned}
\mathscr{R}_{k}^{0, \alpha} & (z, \zeta, R)=\sum_{n=0}^{\infty} e_{k, n}(z) \overline{e_{k, n}(\zeta)} \\
= & \frac{1}{\pi} R^{-2(k+\alpha)} \sum_{n=0}^{\infty} \frac{\Gamma(n+\alpha+1)(k+n+\alpha)}{\Gamma(k) \Gamma(k+\alpha) \Gamma(n+1)} R^{-2 n}\left(R^{2}-|z|^{2}\right)^{-\alpha} \\
& \times \frac{\partial^{k-1}}{\partial z^{k-1}}\left\{\left(R^{2}-z \bar{z}\right)^{k-1+\alpha} z^{n}\right\} \\
& \times \sum_{j=0}^{k-1}(-1)^{k-1-j}\left(\begin{array}{c}
k-1 \\
j
\end{array}\right)\left(\begin{array}{c}
n \\
j
\end{array}\right) j ! \frac{\Gamma(k+\alpha)}{\Gamma(\alpha+j+1)} \bar{\zeta}^{n-j} \zeta^{k-1-j}\left(R^{2}-|\zeta|^{2}\right)^{j}
\end{aligned}
$$

Обозначив сумму по $j$ через $I$, преобразуем ее:

$$
I=\sum_{\nu=0}^{k-1}(-1)^{k-1-\nu} R^{2 \nu}\left(\sum_{j=\nu}^{k-1}\left(\begin{array}{c}
k-1 \\
j
\end{array}\right)\left(\begin{array}{l}
n \\
j
\end{array}\right)\left(\begin{array}{l}
j \\
\nu
\end{array}\right) j ! \frac{\Gamma(k+\alpha)}{\Gamma(\alpha+j+1)}\right) \bar{\zeta}^{n-\nu} \zeta^{k-1-\nu}
$$

Делая подстановку $j-\nu=m$ и воспользовавшись равенством

$$
\left(\begin{array}{c}
k-1 \\
\nu+m
\end{array}\right)\left(\begin{array}{c}
\nu+m \\
\nu
\end{array}\right)=\left(\begin{array}{c}
k-1 \\
\nu
\end{array}\right)\left(\begin{array}{c}
k-1-\nu \\
m
\end{array}\right)
$$

получаем

$$
\begin{aligned}
I= & \sum_{\nu=0}^{k-1}(-1)^{k-1-\nu} R^{2 \nu}\left(\begin{array}{c}
k-1 \\
\nu
\end{array}\right)\left(\sum_{m=0}^{k-1-\nu}\left(\begin{array}{c}
k-1-\nu \\
m
\end{array}\right)\left(\begin{array}{c}
n \\
\nu+m
\end{array}\right)\right. \\
& \left.\times(\nu+m) ! \frac{\Gamma(k+\alpha)}{\Gamma(\alpha+\nu+m+1)}\right) \bar{\zeta}^{n-\nu} \zeta^{k-1-\nu} \\
= & \sum_{\nu=0}^{k-1}(-1)^{k-1-\nu} R^{2 \nu}\left(\begin{array}{c}
k-1 \\
\nu
\end{array}\right)\left(\begin{array}{l}
n \\
\nu
\end{array}\right) \nu !\left(\begin{array}{c}
k-1-\nu \\
\sum_{m=0}^{k-1-\nu} \\
m
\end{array}\right)\left(\begin{array}{c}
n-\nu \\
n-\nu-m
\end{array}\right) \\
& \left.\times m ! \frac{\Gamma(k+\alpha)}{\Gamma(\alpha+\nu+m+1)}\right) \bar{\zeta}^{n-\nu} \zeta^{k-1-\nu} .
\end{aligned}
$$

Рассмотрев функцию $\left(t^{n-\nu} t^{k-1+\alpha}\right)^{(k-1-\nu)}$, как и при доказательстве леммы 3 , легко установить справедливость равенства

$$
\sum_{m=0}^{k-1-\nu}\left(\begin{array}{c}
k-1-\nu \\
m
\end{array}\right)\left(\begin{array}{c}
n-\nu \\
n-\nu-m
\end{array}\right) m ! \frac{\Gamma(k+\alpha)}{\Gamma(\alpha+\nu+m+1)}=\frac{\Gamma(n+k+\alpha-\nu)}{\Gamma(n+\alpha+1)}
$$

Тогда для суммы $I$ окончательно получаем

$$
I=\sum_{\nu=0}^{k-1}(-1)^{k-1-\nu} R^{2 \nu}\left(\begin{array}{c}
k-1 \\
\nu
\end{array}\right)\left(\begin{array}{l}
n \\
\nu
\end{array}\right) \nu ! \frac{\Gamma(n+k+\alpha-\nu)}{\Gamma(n+\alpha+1)} \bar{\zeta}^{n-\nu} \zeta^{k-1-\nu}
$$


Таким образом,

$$
\begin{aligned}
\mathscr{R}_{k}^{0, \alpha}(z, \zeta, R)= & \frac{1}{\pi} R^{-2(k+\alpha)} \sum_{n=0}^{\infty} \frac{\Gamma(n+\alpha+1)(k+n+\alpha)}{\Gamma(k) \Gamma(k+\alpha) \Gamma(n+1)} R^{-2 n}\left(R^{2}-|z|^{2}\right)^{-\alpha} \\
& \times \frac{\partial^{k-1}}{\partial z^{k-1}}\left\{\left(R^{2}-z \bar{z}\right)^{k-1+\alpha} z^{n}\right\} \\
& \times \sum_{\nu=0}^{k-1}(-1)^{k-1-\nu} R^{2 \nu}\left(\begin{array}{c}
k-1 \\
\nu
\end{array}\right)\left(\begin{array}{c}
n \\
\nu
\end{array}\right) \nu ! \frac{\Gamma(n+k+\alpha-\nu)}{\Gamma(n+\alpha+1)} \bar{\zeta}^{n-\nu} \zeta^{k-1-\nu}
\end{aligned}
$$

Отсюда получаем

$$
\begin{aligned}
\psi_{1}^{(s)}(z)= & \frac{1}{\pi} R^{-2(k+s+\alpha)} \frac{\Gamma(k+s+\alpha)(2 k-1+s+\alpha)}{\Gamma(k) \Gamma(k+\alpha)}\left(R^{2}-|z|^{2}\right)^{-\alpha} \\
& \times \frac{\partial^{k-1}}{\partial \bar{z}^{k-1}}\left\{\left(R^{2}-z \bar{z}\right)^{k-1+\alpha} \bar{z}^{k-1+s}\right\}, \quad s=0,1, \ldots, \\
\psi_{2}^{(s)}(z)= & \frac{1}{\pi} R^{-2(k+\alpha)} \frac{2 k-1-s+\alpha}{\Gamma(k-s)}\left(R^{2}-|z|^{2}\right)^{-\alpha} \\
& \times \frac{\partial^{k-1}}{\partial \bar{z}^{k-1}}\left\{\left(R^{2}-z \bar{z}\right)^{k-1+\alpha} \bar{z}^{k-1-s}\right\}, \quad s=0,1, \ldots, k-1 .
\end{aligned}
$$

Найдем теперь нормы функций $\psi_{1}^{(s)}$ и $\psi_{2}^{(s)}$. Для функции $\psi_{1}^{(s)}$, переходя к полярньм координатам, имеем при $1 \leqslant q<\infty$

$$
\begin{aligned}
& \left\|\psi_{1}^{(s)}\right\|_{(q, D(0, R), \alpha)}=\frac{1}{\pi^{1 / p}} R^{-2(k+s+\alpha)} \frac{\Gamma(k+s+\alpha)(2 k-1+s+\alpha)}{\Gamma(k) \Gamma(k+\alpha)} \\
& \quad \times\left\{\int_{0}^{R^{2}}\left(R^{2}-r\right)^{\alpha}\left|r^{-s / 2}\left(R^{2}-r\right)^{-\alpha}\left(\left(R^{2}-r\right)^{k-1+\alpha} r^{k-1+s}\right)^{(k-1)}\right|^{q} d r\right\}^{1 / q} .
\end{aligned}
$$

Сделав подстановку $t=r / R^{2}$, получаем первое равенство леммы 6 . Второе равенство получаем таким же способом, вычислив норму функции $\psi_{2}^{(s)}$. Случай $q=\infty$ рассматриваем аналогично.

ДОКАЗАТЕЛЬСТво ТЕОРЕМЫ 4. Обозначим через $\mathscr{R}_{m}^{\alpha}(\zeta-z, t-z, \rho)$ воспроизводящее ядро для круга $D(z, \rho)$. Тогда, используя теорему 2, получаем

$$
\frac{\partial^{s} f(z)}{\partial z^{s}}=\iint_{D(z, \rho)}\left(\rho^{2}-|\zeta-z|^{2}\right)^{\alpha} f(\zeta) \psi^{(s)}(\zeta-z) d \sigma(\zeta),
$$

где

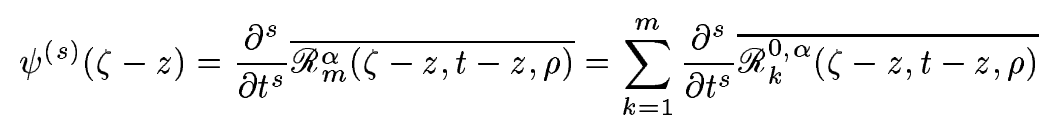

при $t=z$.

Отсюда, применив неравенства Гёльдера и Минковского, а затем лемму 6, получаем первое неравенство. Второе неравенство доказывается аналогично. 
ДокАЗАТЕЛЬСТво теоРемЫ 5. Пусть $f \in \operatorname{Lip}(\beta, p, G), z \in G$. Тогда из теоремы 2 следует, что

$$
\frac{\partial f(z)}{\partial z}=\iint_{D(z, \rho)} f(\zeta) \psi^{(1)}(\zeta-z) d \sigma(\zeta), \quad 0=\iint_{D(z, \rho)} f(z) \psi^{(1)}(\zeta-z) d \sigma(\zeta) .
$$

Отсюда имеем

$$
\begin{aligned}
\left|\frac{\partial f(z)}{\partial z}\right| & \leqslant \iint_{D(z, \rho)}|f(\zeta)-f(z)|\left|\psi^{(1)}(\zeta-z)\right| d \sigma(\zeta) \\
& \leqslant\left\{\iint_{D(z, \rho)}|f(\zeta)-f(z)|^{p} d \sigma(\zeta)\right\}^{1 / p}\left\{\iint_{D(z, \rho)}\left|\psi^{(1)}(\zeta-z)\right|^{q} d \sigma(\zeta)\right\}^{1 / q} \\
& \leqslant C \rho^{\beta}\left\{\iint_{D(z, \rho)}\left|\psi^{(1)}(\zeta-z)\right|^{q} d \sigma(\zeta)\right\}^{1 / q} .
\end{aligned}
$$

Остается применить лемму 6 при $\alpha=0$.

Оценку для $|\partial f / \partial \bar{z}|$ получаем аналогично.

Докажем теперь обратное утверждение. Пусть

$$
\left|\frac{\partial f(z)}{\partial z}\right|+\left|\frac{\partial f(z)}{\partial \bar{z}}\right|=O\left(\rho^{\beta-1-2 / p}\right) \quad \text { при } \rho=\rho(z, \partial G) \rightarrow 0 .
$$

Если $\zeta \in D(z, R), R<\rho$, то интегрируя по отрезку с концами в точках $z$ и $\zeta$, получаем

$$
f(\zeta)-f(z)=\int_{0}^{1}\left(\frac{\partial f}{\partial z}(\zeta-z)+\frac{\partial f}{\partial \bar{z}} \overline{(\zeta-z)}\right) d \theta
$$

где $\partial f / \partial z$ и $\partial f / \partial \bar{z}$ вычислены в точке $t=z+\theta(\zeta-z), 0 \leqslant \theta \leqslant 1$.

Из равенства (19), применив обобщенное неравенство Минковского и сделав замену переменных, получаем

$$
\|f(\cdot)-f(z)\|_{(p, D(z, R))} \leqslant \int_{0}^{1} \theta^{-\frac{2}{p}-1}\left\{\iint_{D(z, \theta R)}\left|\frac{\partial f}{\partial z}(t-z)+\frac{\partial f}{\partial \bar{z}} \overline{(t-z)}\right|^{p} d \sigma(t)\right\}^{1 / p} d \theta,
$$

где $\partial f / \partial z$ и $\partial f / \partial \bar{z}$ вычислены в точке $t \in D(z, \theta R), 0 \leqslant \theta \leqslant 1$.

Обозначим через $\rho_{t}$ расстояние от точки $t \in D(z, \theta R)$ до $\partial G$. Очевидно, что

$$
\rho_{t} \geqslant \rho(z, \partial G)-|t-z|=\rho-|t-z| .
$$

Тогда, учитьвая (18) и переходя к полярньм координатам, из (20) получаем

$$
\|f(\cdot)-f(z)\|_{(p, D(z, R))} \leqslant C \rho^{\beta}\left(\frac{R}{\rho}\right)^{1+\frac{1}{p}} \int_{0}^{1} \theta^{-\frac{1}{p}}\left\{\int_{0}^{\frac{\theta R}{\rho}}(1-r)^{(\beta-1) p-2} d r\right\}^{1 / p} d \theta .
$$

Так как $\beta>1 / p$, то из последнего неравенства получаем требуемое. 


\section{СПИСОК ЦИТИРОВАННОЙ ЛИТЕРАТУРЫ}

[1] Balk M. B. Polyanalytic Functions. Berlin: Akad. Verlag, 1991.

[2] Долженко Е. П. О граничном поведении компонент полианалитической функции // Докл. РАН. 1994. Т. 338. № 5. С. 585-588.

[3] Долженко Е. П. О граничном поведении компонент полианалитической функции // Матем. заметки. 1998. Т. 63. №6. С. 821-834.

[4] Долженко Е. П., Данченко В. И. О граничном поведении решений обобщенного уравнения Коши-Римана // Вестн. МГУ. Сер. 1. Матем., мех. 1998. № 3. С. 16-25.

[5] Рамазанов А.К. Об ортогональных проекторах в пространствах полианалитических функций // Изв. Тульского гос. ун-та. Сер. матем., мех., информатика. 1998. Т. 4. № 1. C. $117-122$.

[6] Рамазанов А.К. Представление пространства полианалитических функций в виде прямой суммы ортогональных подпространств. Приложение к рациональным аппроксимациям // Матем. заметки. 1999. Т. 66. № 5. С. 741-759.

[7] Долженко Е. П., Данченко В. И. О граничном поведении производных полианалитических функций // Современные проблемы теории функций. Тезисы докл. 9-й Саратовской зимней школы. Саратов, 1997. С. 54.

[8] Захарюта В.П., Юдович В.И. Общий вид линейного функционала в $H_{p}^{\prime} / /$ УМН. 1964. T. 19. № 2. C. 139-142.

[9] Burbea J. The Bergman projection over plane regions // Ark. Mat. 1980. V. 18. № 2. P. 207-221.

[10] Соловьев А. А. Оценки в $L_{p}$ интегральных операторов, связанных с пространствами аналитических и гармонических функций // Сиб. матем. ж. 1985. Т. 26. № 3. С. 168-191.

[11] Васин А. В. Проекторы на $L^{p}$-пространства полианалитических функций // Зап. научн. семинаров ЛОМИ. 1991. Т. 190. С. 15-33.

[12] Кошелев А. Д. О керн-функции для гильбертова пространства полианалитических функций в круге // Докл. АН СССР. 1977. Т. 232. № 2. С. 277-279.

[13] Вячеславов Н. С., Рамазанов А. К. Интерполяционные свойства рациональных функций наилучшего приближения в среднем квадратическом на окружности и в круге // Матем. заметки. 1995. Т. 57. №2. С. 228-239.

[14] Duren P. L. Theory of $H^{p}$ Spaces. New York: Academic Press, 1970.

[15] Забулёнис А. О дифференциальном операторе в пространствах аналитических функций // Литовский матем. ж. 1984. Т. 24. №1. С. 53-58.

[16] Рудин У. Теория функций в единичном шаре из $\mathscr{C}^{n}$. М., 1984.

Калужский филиал МГТУ им. Н. Э. Баумана

Поступило

13.02.2001

Исправленный вариант

16.10.2001 